\title{
Surface of Strontium Titanate
}

\author{
R. Herger, P. R. Willmott, ${ }^{*}$ O. Bunk, C. M. Schlepütz, and B. D. Patterson \\ Swiss Light Source, Paul Scherrer Institut, CH-5232 Villigen, Switzerland \\ B. Delley \\ Condensed Matter Theory Group, Paul Scherrer Institut, CH-5232 Villigen, Switzerland
}

(Received 22 November 2006; published 15 February 2007)

\begin{abstract}
We report the first complete determination, using surface $\mathrm{x}$-ray diffraction, of the surface structure of $\mathrm{TiO}_{2}$-terminated $\mathrm{SrTiO}_{3}(001)$, both at room temperature in vacuum, and also hot, under typical conditions used for thin film growth. The cold structure consists of a mixture of a $(1 \times 1)$ relaxation and $(2 \times 1)$ and $(2 \times 2)$ reconstructions. The latter disappear over several minutes upon heating. The structures are best modeled by a $\mathrm{TiO}_{2}$-rich surface similar to that proposed by Erdman et al. [Nature (London) 419, 55 (2002).]. Both reconstructions have been shown by density functional theory to be energetically favorable. The calculated $(1 \times 1)$ surface energy is higher, indicating that it may be a disordered mixture of the reconstructions. Atomic displacements are significant down to three unit cells, which may have important implications on possible surface ferroelectric phenomena in $\mathrm{SrTiO}_{3}$.
\end{abstract}

DOI: 10.1103/PhysRevLett.98.076102

Strontium titanate $\left(\mathrm{SrTiO}_{3}, \mathrm{STO}\right)$ is the paradigmatic substrate material for epitaxial growth of thin films of the technologically important family of perovskites, including high-temperature superconductors, colossal magnetoresistive materials, ferroelectrics, and heterostructures containing two-dimensional electron gases [1-4]. The surface structure of STO has therefore been the subject of intense research in recent years and a detailed description of the surface with sub- $\AA$ resolution is hence of great importance. Despite this effort, the surface structure of STO remains a subject of controversy, due on the one hand to its sensitivity to the preparation and processing conditions, and on the other, to the limited spatial resolution and chemical sensitivity associated with most surface characterization techniques. According to preparation and ambient conditions, at least 8 different reconstructions and relaxations have been reported for the STO(001) surface alone [5-14].

Among these, the unusual $(2 \times 1)$ reconstruction proposed by Erdman et al. [5] has provoked much debate $[9,11,12,14,15]$. In this model, the surface terminates not with one, but two $\mathrm{TiO}_{2}$ layers, i.e., the surface is Ti rich, while the topmost Ti atoms cluster to form a "zigzag" motif. Such structural distortions and rearrangements could affect the nucleation mechanisms in thin film growth and may also induce different physical properties from those of the bulk, including ferroelectricity $[2,16]$, catalytic behavior, and spin-polarization/double exchange mechanisms at thin film interfaces to manganites [17].

For thin film growth, a reliable chemical and thermal preparation of the STO substrate surface has become established, which ensures $100 \%$ termination on the $\mathrm{TiO}_{2}$ sublayer (SL) and smooth terrace edge profiles [18-20]. In this Letter we present the temperature-dependent structure of STO(001) prepared in this standard manner, first at room temperature ("cold") and in ultrahigh vacuum (UHV), and, subsequently, under conditions typical for perovskite
PACS numbers: 68.35.Bs, 61.10.Nz, 71.15.Mb, 77.84.Bw

thin film growth (heated to $750 \pm 30^{\circ} \mathrm{C}$ in $10^{-3} \mathrm{~Pa} \mathrm{O}_{2}-$ "hot"). The structures were determined using surface $\mathrm{x}$-ray diffraction (SXRD) [21,22], which is one of only a few techniques capable of providing the sub- $\AA$ resolution necessary to predict the detailed physical properties of crystalline surfaces. The different surface structures presented here were found to all exhibit Ti clustering and a surface $\mathrm{TiO}_{2}$ double layer.

The morphology of the prepared STO substrate surface was checked using atomic force microscopy (AFM) and found to consist only of straightedged terraces of $0.4 \mathrm{~nm}$ height (i.e., one unit cell) and $250 \mathrm{~nm}$ width, while $\mathrm{x}$-ray photoelectron spectroscopy confirmed termination with $\mathrm{TiO}_{2}$. Substrates were then introduced in a UHV chamber equipped with a large beryllium window [23] mounted on a $(2+3)$ circle surface diffractometer at the materials science beam line of the Swiss Light Source, Paul Scherrer Institut. Thanks to the availability of a fast x-ray photoncounting pixel detector [24], it was feasible to reliably record the large data set of independent structure factors necessary to obtain a convincing model within the limited allocated beam time. Two independent sets of data from two different samples were recorded with $1 \AA$ synchrotron radiation for both the cold and hot conditions, to confirm reproducibility.

In addition to crystal truncation rods (CTRs), superstructure rods $(\mathrm{SSRs})$ associated with $(2 \times 2)$ and $(2 \times 1)$ surface reconstructions were identified in the SXRD signal for the cold sample. These SSR and CTR signals were stable in the cold conditions over the measurement time of over $24 \mathrm{~h}$, though the SSRs vanished over several minutes when the substrate was brought to the hot conditions [25].

A representative subset of the cold data is shown in Fig. 1(b), along with the error bars, which derive primarily from systematic differences between symmetry-equivalent 
structure factors. The complete data set consists of 9 CTRs and 18 SSRs, totaling 1668 nonequivalent structure factors, (plus 806 equivalent data points) and spans $|h|,|k|$, and $l=$ 0 to 3 . The fits were generated by the program FIT [26] using 394 parameters (i.e., an oversampling factor of 4). These included atomic coordinates, Debye-Waller (DW) factors, and fractional contributions from each surfacestructure type. Although the final goodness of fit is given in terms of the crystallographic $R$ factor, fit optimization was via $\chi^{2}$-minimization, in order to avoid artificial weighting of the most intense signal near the Bragg maxima in the CTRs [27].

The structure comprises all 3 surface domains shown in Fig. 1(a), including their symmetry-equivalent orientations, and significant relaxations are observed down to a depth of 3 unit cells. The final model, shown in Fig. 1(c), has a crystallographic $R$ factor of 4.5\% [28] and no unphysical positions or DW factors. It is dominated by the $(2 \times 2)(43 \%)$ and $(2 \times 1)(37 \%)$ reconstructions, plus a smaller contribution $(20 \%)$ from a $(1 \times 1)$ relaxation.

The model shown in Fig. 1 was only one of 50 that were tested for the cold data, including selected models from the literature $[5,6,9,11,13,29,30]$. In arriving at this model, we were guided by clear and consistent trends: all models which contained both the surface $\mathrm{TiO}_{2}$ double layer (DL) and a zigzag motif of the top Ti-atoms in the $(2 \times 1)$ and $(2 \times 2)[5,14,30]$ produced significantly better fits than other models. A detailed description of all investigated models will be given elsewhere [31].

The SXRD data of STO(001), recorded under the hot conditions, consisted of 837 structure factors plus 764 equivalent data points ( $|h|,|k|$, and $l=0$ to 3 ) and showed no SSRs. The data was fit with 57 atomic positional and DW parameters, i.e., an oversampling factor of more than 10. Seven models were tested. The best final surface structure contained no unphysical parameters and is shown in Fig. 2. It has a crystallographic $R$ factor of $11.2 \%$. It is very similar to the $\mathrm{DL}(1 \times 1)$ structure for the cold data, but with more puckering of the top $\mathrm{TiO}_{2} \mathrm{SL}$, as the topmost $\mathrm{Ti}$ atom moves further out from the surface.

The physical correctness of the models is supported on the one hand by previous experimental evidence regarding the DL $(2 \times 1)$ domain [5], but also by theoretical calculations, which predicted the low surface energy of STO(001) terminated in much the same manner as the DL $(2 \times 2)$ described here [30]. Indeed, our own density functional theory (DFT) calculations [32,33] using the PBE functional [34] have shown that the three lowest surface energy configurations of STO that were tested include the two reconstructions we have found experimentally (see Table I). The DL $(2 \times 2)$ surface can be considered as consisting of alternately flipped DL $(2 \times 1)$ surface cells, and hence their similar chemistry explains their comparable surface energies.

The other low-energy configuration is the bulk $(1 \times 1)$ surface (i.e., that without the $\mathrm{TiO}_{2}$ overlayer). Fits of the data using this bulk $(1 \times 1)$ termination produced significantly poorer results. Also, this surface would be half a unit cell lower or higher $( \pm 0.2 \mathrm{~nm})$ than adjacent DL reconstructions, and yet we see no evidence of this in the AFM images. It has been suggested in the literature that such "ideal" bulk $(1 \times 1)$ surfaces do not exist in practice, due to surface oxygen vacancies [12]. Although we have no evidence of such vacancies from our fits, the slightly enlarged Debye-Waller factors of the surface oxygen atoms may reflect less than unity occupation.

Transformation between the DL $(1 \times 1)$ relaxation and either reconstruction only requires a diagonal hop of every second Ti-atom across half a surface $(1 \times 1)$ unit cell. However, the surface energy of the $\mathrm{TiO}_{2} \mathrm{DL}(1 \times 1)$ seems (a)

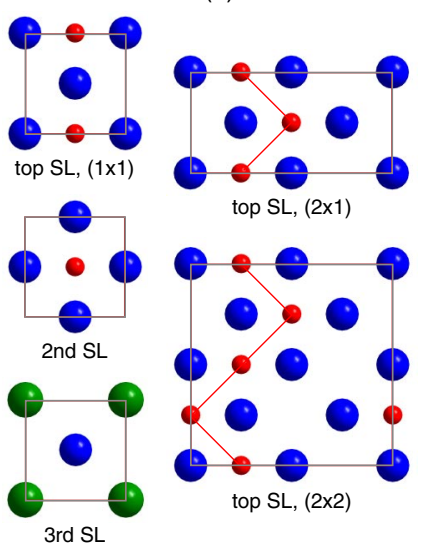

(b)

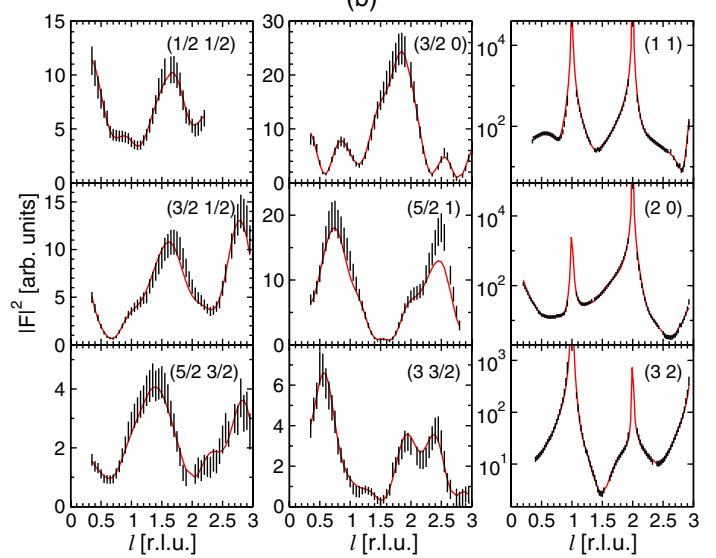

(c)

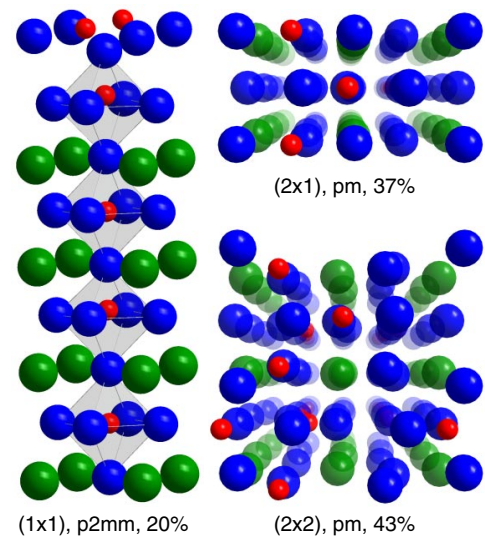

FIG. 1 (color online). The surface structure of cold STO: (a) Starting models of the 3 different domains. SL $=$ sublayer. The 2nd SL $\left(\mathrm{TiO}_{2}\right)$ and $3 \mathrm{rd} \mathrm{SL}(\mathrm{SrO})$ together make up a bulk STO unit cell. Note the zigzag motifs in the $(2 \times 1)$ and $(2 \times 2)$ reconstructions highlighted in red. (b) Subset of the SXRD data (black) and fits (red). We have included the (5/2 1l) SSR, which shows the largest deviations between the fit and experimental data for the entire set. (c) The final models for the three domains, including their symmetries and percentage contributions. The $(1 \times 1)$ structure is viewed from the side, while the reconstructions are from above. Blue, O; red, Ti; green, $\mathrm{Sr}$. 
TABLE I. Surface energies in $\mathrm{eV} /(1 \times 1)$ unit cell (with respect to the SrO chemical potential) of 4 domain types calculated using DFT. The bulk $(1 \times 1)$ model does not contain the extra $\mathrm{TiO}_{2}$ sublayer shown upper left in Fig. 1(a).

\begin{tabular}{cccc}
\hline \hline Bulk $(1 \times 1)$ & DL $(1 \times 1)$ & DL $(2 \times 1)$ & DL $(2 \times 2)$ \\
\hline 0.78 & 1.39 & 0.78 & 0.38 \\
\hline \hline
\end{tabular}

anomalously high (Table I). The presence, in spite of this, of a $(1 \times 1)$ structure could have two reasons: either this domain is metastable with a significant activation barrier to lower-energy states, or in fact consists of a (possibly dynamic) disordered matrix of the zigzag motif of the $(2 \times 1)$ and $(2 \times 2)$, randomly flipped and mirrored [35]. This disordered $(1 \times 1)$ domain would have a surface energy comparably low to those of the two reconstructions, and the average structure is adequately described by the $(1 \times 1)$ structure shown in Fig. 1. Such a model would not have to invoke coordinated and concerted Ti hopping across the surface over large distances (of the order of $100 \mathrm{~nm}$ ) to go from the reconstructions to the $(1 \times 1)$ relaxation. Disorder could also explain how such a $(1 \times 1)$ surface can dominate at elevated temperatures, where the surface thermal vibrational energy becomes comparable to the activation barrier between the $(2 \times 1)$ and $(2 \times 2)$, leading to a disordered mixture of these. Indeed, the difference in surface energy between two $(2 \times 1)$ cells and a single $(2 \times 2)$ cell is $\Delta E_{s}=1.6 \mathrm{eV}$ (see Table I). On the other hand, the vibrational energy of the surface atoms of the same system is, to a first approximation, equal to $E_{v}=3 k T\left(4 n_{a}\right)$, where $k$ is Boltzmann's constant, $T$ is the temperature, $n_{a}$ is the number of atoms per top sublayer and unit cell (here, for $\mathrm{TiO}_{2}, n_{a}=3$ ), and the factor 4 accounts for the fact that 4
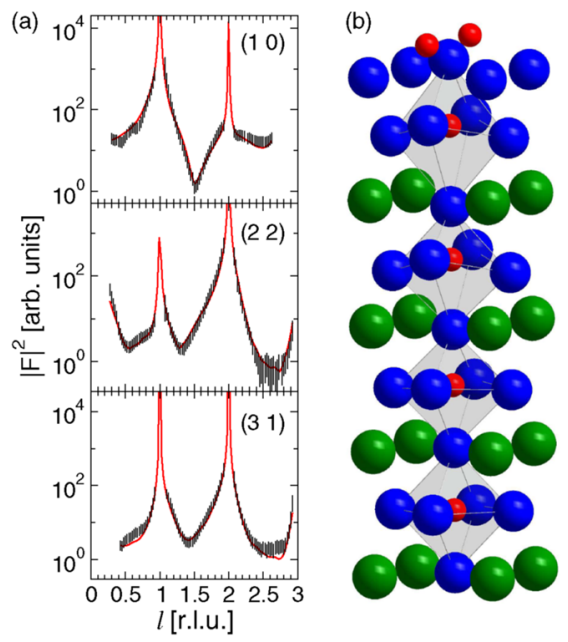

FIG. 2 (color online). The surface structure of $(1 \times 1)$ hot STO: (a) Subset of three rods from a total of nine of the SXRD data (black) and their fits (red), based on the same starting model as the DL $(1 \times 1)$ domain for the cold data shown in Fig. 1(a). (b) The final model for the hot DL $(1 \times 1)$ surface ( $p 2 \mathrm{~mm}$ symmetry). Color code as in Fig. 1. surface $(1 \times 1)$ cells are needed to describe this system. We therefore obtain $E_{v}=3.2 \mathrm{eV}$ under hot conditions, i.e., twice the difference in reconstructions' surface energies. The size of the activation barrier between the two reconstructions will affect the time needed to reach this mixed equilibrium state. From the Arrhenius rate constant of the disappearance of the SSR signal of the order of $k_{s}=$ $0.01 \mathrm{~s}^{-1}$ and assuming a preexponential factor $A \sim 10^{12}$, we obtain an activation energy of $E_{a} \approx 3 \mathrm{eV}$. For the room temperature sample, $E_{v}=0.9 \mathrm{eV}$ and the reaction rate constant is of the order of $10^{-40} \mathrm{~s}^{-1}$; i.e., the system is completely kinetically hindered [36].

A further argument supporting the DL model is based on the electrostatic requirements of polar surfaces. The partially covalent nature of the bonds in STO results in the sublayers of the bulk ( $\mathrm{TiO}_{2}$ and $\left.\mathrm{SrO}\right)$ having nonzero and opposite net charges $\pm \sigma$ [37]. For such polar systems, the top sublayer of stable surfaces are required to have a charge of $\sigma / 2$, in order to avoid a physically unreasonable linear increase in the electric field with depth into the crystal. This condition is met by the top $\mathrm{TiO}_{2}$ sublayer in bulkterminated $\mathrm{STO}(001)$, which differs from lower $\mathrm{TiO}_{2}$ sublayers by having a reduced number of bonds. The addition of the extra $\mathrm{TiO}_{2}$ sublayer in the DL model modifies the charge of the second $\mathrm{TiO}_{2}$ sublayer. However, simple calculations show that this $\mathrm{TiO}_{2}$ overlayer in all three proposed DL surface structures compensates for this change and satisfies the criterion of electrostatic compensation.

Displacements, $\Delta z$, of the atomic positions in the final models compared to the high-symmetry positions in the starting models are shown in Fig. 3 in the direction of the sample normal, as it is in this direction that they are most prominent. These data, derived from experimental results, are very similar in trend to those theoretically predicted by Johnston et al. [12], with the uppermost atoms on average

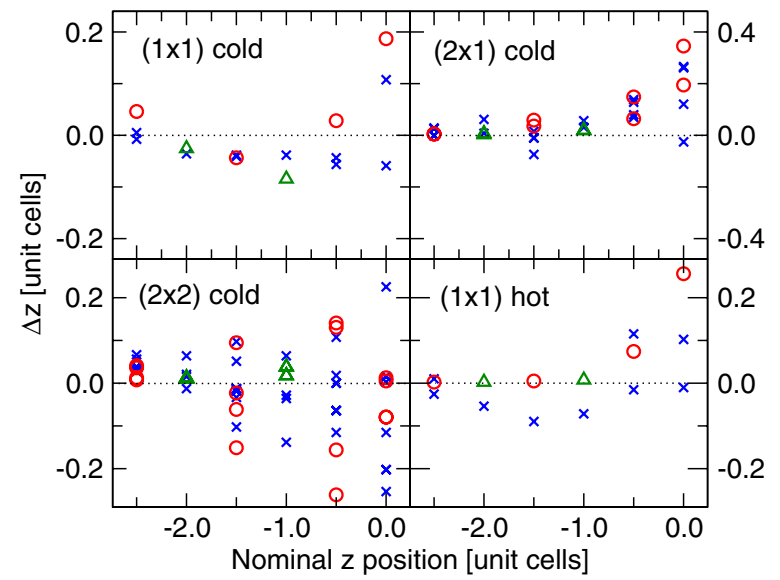

FIG. 3 (color online). Displacements of $\mathrm{Sr}$ (green triangles), Ti (red circles), and $\mathrm{O}$ (blue crosses) in the $z$ direction (i.e., normal to the surface) from the high-symmetry positions in the starting models, as a function of $z$ for the proposed cold and hot models. The nominal surface is at $z=0$ and positive values of $\Delta z$ indicate displacements towards the vacuum. 
displaced outwards to the vacuum. The loss of centrosymmetry of the $\mathrm{Ti}$ atoms within the oxygen octahedra may lead to a permanent surface dipole moment and surface ferroelectricity $[2,16,38]$. Indeed, surface polarity is tacitly implied in the electrostatic arguments given above.

In conclusion, we have solved the surface structure of the scientifically and technologically important perovskite material $\mathrm{SrTiO}_{3}(001)$ using surface x-ray diffraction, under optimal preparation and typical thin film growth conditions. For the first time, 3 structures simultaneously present on the same surface could be refined from a single data set. All three structures contain a characteristic double $\mathrm{TiO}_{2}$ top layer, while the two reconstructions, predicted by DFT calculations to be energetically favorable, are formed by repetition of a common zigzag motif. It is suggested that the $(1 \times 1)$ structure may be an energetically favorable, disordered mixture of the two reconstructions. This would help explain the presence of only the $(1 \times 1)$ structure upon heating the sample, as the surface vibrational energy exceeds the difference in surface energy between the $(2 \times 1)$ and $(2 \times 2)$ domains, resulting in their complete mixing. The final models exhibit significant deviations from their high-symmetry starting positions down to a depth of 3 unit cells, which may have important consequences on the surface's ferroelectric and other nonlinear properties. Finally, it is hoped that theoretical calculations based on these models will provide a deeper insight into the physics of this fascinating surface.

We thank D. K. Saldin, P. F. Lyman, and V. L. Shneerson for fruitful discussions. Support of this work by the Schweizerischer Nationalfonds zur Förderung der wissenschaftlichen Forschung and the staff of the Swiss Light Source is gratefully acknowledged. This work was performed at the Swiss Light Source, Paul Scherrer Institut.

*Electronic address: philip.willmott@psi.ch

[1] S. Jin, T.H. Tiefel, M. McCormack, R. A. Fastnacht, R. Ramesh, and L. H. Chen, Science 264, 413 (1994).

[2] D. D. Fong, G. B. Stephenson, S. K. Streiffer, J. A. Eastman, O. Auciello, P. H. Fuoss, and C. Thompson, Science 304, 1650 (2004).

[3] C. H. Ahn, K. M. Rabe, and J.-M. Triscone, Science 303, 488 (2004).

[4] A. Ohtomo and H. Y. Hwang, Nature (London) 427, 423 (2004).

[5] N. Erdman, K. R. Poeppelmeier, O. Warschkow, D. E. Ellis, and L. D. Marks, Nature (London) 419, 55 (2002).

[6] T. Matsumoto, H. Tanaka, T. Kawai, and S. Kawai, Surf. Sci. 278, L153 (1992).

[7] Q. D. Jiang and J. Zegenhagen, Surf. Sci. 425, 343 (1999).

[8] G. Charlton, S. Brennan, C. A. Muryn, R. McGrath, D. Norman, T. S. Turner, and G. Thornton, Surf. Sci. 457, L376 (2000).

[9] T. Kubo and H. Nozoye, Phys. Rev. Lett. 86, 1801 (2001).

[10] A. Kazimirov, D. M. Goodner, M. J. Bedzyk, J. Bai, and C. R. Hubbard, Surf. Sci. 492, L711 (2001).

[11] M. R. Castell, Surf. Sci. 505, 1 (2002).
[12] K. Johnston, M. R. Castell, A. T. Paxton, and M. W. Finnis, Phys. Rev. B 70, 085415 (2004).

[13] V. Vonk, S. Konings, G. J. van Hummel, S. Harkema, and H. Graafsma, Surf. Sci. 595, 183 (2005).

[14] F. Silly, D. T. Newell, and M. R. Castell, Surf. Sci. 600, L219 (2006).

[15] L. M. Liborio, C. G. Sánchez, A. T. Paxton, and M.W. Finnis, J. Phys. Condens. Matter 17, L223 (2005).

[16] N. Bickel, G. Schmidt, K. Heinz, and K. Müller, Phys. Rev. Lett. 62, 2009 (1989).

[17] V. Garcia, M. Bibes, A. Barthélémy, M. Bowen, E. Jacquet, J.-P. Contour, and A. Fert, Phys. Rev. B 69, 052403 (2004).

[18] M. Kawasaki, K. Takahashi, T. Maeda, R. Tsuchiya, M. Shinohara, O. Ishiyama, T. Yonezawa, M. Yoshimoto, and H. Koinuma, Science 266, 1540 (1994).

[19] G. Koster, B. L. Kropman, G. J.H. M. Rijnders, D. H. A. Blank, and H. Rogalla, Appl. Phys. Lett. 73, 2920 (1998).

[20] T. Ohnishi, K. Shibuya, M. Lippmaa, D. Kobayashi, H. Kumigashira, M. Oshima, and H. Koinuma, Appl. Phys. Lett. 85, 272 (2004).

[21] R. Feidenhans'l, Surf. Sci. Rep. 10, 105 (1989).

[22] I. K. Robinson and D. J. Tweet, Rep. Prog. Phys. 55, 599 (1992).

[23] P. R. Willmott, C. M. Schlepütz, B. D. Patterson, R. Herger, M. Lange, D. Meister, D. Maden, C. Brönnimann, E. F. Eikenberry, and G. Hülsen et al., Appl. Surf. Sci. 247, 188 (2005).

[24] C. M. Schlepütz, R. Herger, P. R. Willmott, B. D. Patterson, O. Bunk, C. Brönnimann, B. Henrich, G. Hülsen, and E. F. Eikenberry, Acta Crystallogr. Sect. A 61, 418 (2005).

[25] Note also, that all evidence of reconstructions vanished after exposure of the surface to less than $1 \mathrm{~s}$ (approximately 0.1 monolayer) of flux from hyperthermal particles having kinetic energies of the order of $25 \mathrm{eV}$ during pulsed laser deposition.

[26] O. Bunk, Ph.D. thesis, University of Hamburg, 1999, URL http://www.sub.uni-hamburg.de/opus/volltexte/1999/99/.

[27] Despite this, exchanging the goodness-of-fit criterion from $\chi^{2}$ to minimization of the crystallographic $R$ factor and back led to the same final model.

[28] W. C. Hamilton, Acta Crystallogr. 18, 502 (1965).

[29] Q. D. Jiang and J.Zegenhagen, Surf. Sci. 338, L882 (1995).

[30] O. Warschkow, M. Asta, N. Erdman, K. R. Poeppelmeier, D. E. Ellis, and L. D. Marks, Surf. Sci. 573, 446 (2004).

[31] R. Herger, P. R. Willmott, O. Bunk, C. M. Schlepütz, B. D. Patterson, and B. Delley (to be published).

[32] B. Delley, J. Chem. Phys. 92, 508 (1990).

[33] B. Delley, J. Chem. Phys. 113, 7756 (2000).

[34] J. P. Perdew, K. Burke, and M. Emzerhof, Phys. Rev. Lett. 77, 3865 (1996).

[35] L. D. Roelofs, G. Y. Hu, and S. C. Ying, Phys. Rev. B 28, 6369 (1983).

[36] On a speculative note, the disordered $(1 \times 1)$ domains suggested here may, in the cold sample, comprise regions, or "grain boundaries" of disorder between the ordered $(2 \times$ $1)$ and $(2 \times 2)$ reconstructions, frozen in as the sample slowly cools at the end of the substrate preparation procedure.

[37] C. Noguera, J. Phys. Condens. Matter 12, R367 (2000).

[38] V. Ravikumar, D. Wolf, and V. P. Dravid, Phys. Rev. Lett. 74, 960 (1995). 